\title{
Bullous Keratopathy in a Dog Treated with Third Eyelid Flap and Autologous Serum Eye Drops
}

\author{
Danilo Rocha de Melo', Jaine Mendes Lopes', Ana Karoline Rodrigues da Costa ', Jéssica Fontes Veloso', \\ Renata Santiago Alberto Carlos², Layze Cilmara Alves da Silva Vieira', \\ Wagner Rocha de Souza' \& Deusdete Conceição Gomes Junior ${ }^{1}$
}

\begin{abstract}
Background: Bullous Keratopathy (BK) is characterized by decompensation of the corneal stroma resulting in visual impairment by corneal opacity, discomfort, excessive tearing, blepharospasm and pain. The aim of this study was to report a case of BK in a dog and the complete recovery of the ocular structure and visual function, with a third eyelid flap associated with the use of autologous blood serum topically.

Case: A 2-year-old Shih Tzu male dog, weighing $4.3 \mathrm{~kg}$, with recurrent bilateral eye discomfort was brought to Ophthalmologist Veterinarian Assistance. The patient had already been seen by other veterinarians, but the signs returned in a short period of time after the prescription was suspended. During physical examination it was observed moderate blepharospasm in the right eye, epiphora in the left eye, and in both eyes was noted exophthalmos, medial corneal entropion, caruncle trichiasis and ectopic cilia in the upper and lower eyelids. Fluorescein test was positive in both eyes, and a large bullous, opaque and gelatinous lesion with irregular appearance was observed occupying a large part of the cornea of the right eye. Superficial corneal ulcer was diagnosed in left eye and BK in right eye, both probably in consequence of ectopic cilia presence. Surgical intervention was made, with the cauterization and excision of the ectopic cilia and third eyelid flap in the right eye. The cauterization of caruncle trichiasis was not authorized by the owner. Moxifloxacin eye drops in the dose of one drop four times a day in both eyes, lubricant based on hyaluronic acid in the dose of one drop four times a day in both eyes, and autogenous blood serum, obtained from the centrifugation of a blood sample of the dog itself, in the dose of one drop every hour in the right eye for $24 \mathrm{~h}$, and meloxicam in the dose of $0.1 \mathrm{mg} / \mathrm{kg}$ every $24 \mathrm{~h}$ for 3 days were prescribed. Elizabethan collar was recommended until removal of the third eye flap. During the surgery, a sample of eye secretion was collected and sent for culture and antibiogram and did not show growth of microorganisms. After seven days of the procedure, the animal did not show eye discomfort and corneal ulcer in the left eye was healed. The third eye flap remained stable until the return of 28 days and after removal of the capton, adhesions were identified, which were removed with Castroviejo scissors after application of topical anesthetic proxymetacaine hydrochloride eye drops. A fluorescein test was performed and the absence of corneal keratitis was confirmed. Discrete corneal scars (macula) were identified.

Discussion: The patient in this report had several changes in ocular morphology that favored the formation of recurrent injuries which contributed to the formation of BK. The use of topical medications as unique treatment was not performed due to the severity of the lesion and the presence of alterations such as medial entropion, caruncle trichiasis and ectopic cilia that needed surgical corrections to resolve the causes of the lesions. In order to protect the cornea and provide compression of its epithelium, a third eyelid flap was performed, a low-invasive procedure that is quick and easy to perform. Ectopic cilia were cauterized to eliminate one of the causes of the corneal injuries, however, the other changes could not be corrected because they were not authorized by the owner. The adjuvant uses of ocular lubricant based on hyaluronic acid, a broad-spectrum topical antibiotic and mainly autogenous blood serum favored the epithelial reconstruction of the cornea by providing protection, vitamins, growth factors, immunoglobulins, anti-collagenolytic and bacteriostatic substances. The protocol used it enabled a rapid reduction in patient discomfort, reversal of injuries and recovery of visual function.
\end{abstract}

Keywords: eye disease, cornea, corneal dystrophy.

DOI: $10.22456 / 1679-9216.102854$

Received: 12 May 2020

Accepted: 20 0ctober 2020

Published: 18 November 2020

${ }^{1}$ Multidisciplinary Campus of Barra, Federal University of Western Bahia (UFOB), Barra, BA, Brazil. ${ }^{2}$ Small Animal Clinic, Department of Agrarian and Environmental Sciences, Veterinary Medicine Course, State University of Santa Cruz (UESC), Ilhéus, BA. CORRESPONDENCE: J.F. Veloso [jessica. veloso@ ufob.edu.br]. Federal University of Western Bahia, Multidisciplinary Campus of Barra. Av. 23 de Agosto n. 872. CEP 47100-000 Barra, BA, Brazil. 


\section{INTRODUCTION}

Bullous Keratopathy (BK) is an acquired alteration characterized by decompensation of the corneal stroma, caused by proteolytic enzymes arising from inflammatory cells, pathogens or dysfunction of the cells. It is reported in dogs and cats, and the brachycephalic breeds are the most affected $[4,10]$. BK is characterized by decompensation of the corneal stroma resulting in visual impairment by corneal opacity, discomfort, excessive tearing, blepharospasm and pain $[4,6,8,10]$.

The diagnosis is based on ophthalmic examination findings and treatment can be clinical or surgical. In clinical treatment, hyperosmotic solutions associated with topical antibiotics and anti-inflammatory drugs are recommended. Surgical treatments include thermokeratoplasty, conjunctival flap, deep lamellar keratoplasty, tarsorrhaphy, amniotic membrane transplantation, ultraviolet-A collagen cross-linking and third eyelid flap $[8,10]$.

The aim of this study was to report a case of BK in a dog and the complete recovery of the ocular structure and visual function, with a third eyelid flap associated with the use of autologous blood serum topically.

\section{CASE}

A 2-year-old Shih Tzu male dog, weighing 4.3 $\mathrm{kg}$, with recurrent bilateral eye discomfort was brought to the Ophthalmic Service of the Federal University of Western Bahia (UFOB). The patient had already been seen by 4 other veterinarians and was prescribed for both eyes drugs based on Gatifloxacin ${ }^{1}$, Moxifloxacin ${ }^{2}$ and lubricants based on Dextran ${ }^{3}$ and Hypromello$\mathrm{se}^{4}$. According to the owner, clinical improvement occurred, but when the treatment was suspended, the ophthalmic signs returned in a short period of time. It was also reported that in the last veterinary care (4 days before) a topical Diclofenac sodium ${ }^{1}$ in the dose of one drop every $8 \mathrm{~h}$ in both eyes was added, and after this, there was a quickly reduction in conjunctival hyperemia, however, the cornea of the right eye became opaque and with a gelatinous appearance.

During physical examination, the animal was alert, with physiological parameters within the normal range for the species. In the ophthalmic examination, both eyes and periocular region were examined in pursuit of gross abnormalities with a $3 \mathrm{X}$ magnification binocular loupe and a transilluminator.
Moderate blepharospasm was observed in the right eye, epiphora in the left eye, and in both eyes was noted exophthalmos, medial corneal entropion, caruncle trichiasis and ectopic cilia in the upper and lower eyelids. Schirmer tear test (STT) was $19 \mathrm{~mm}$ in the left eye and $17 \mathrm{~mm}$ in the right eye. Fluorescein test was positive in both eyes, and a large bullous, opaque and gelatinous lesion with irregular appearance was observed occupying a large part of the cornea of the right eye (Figure 1A). Superficial corneal ulcer was diagnosed in the left eye and BK in the right eye, both probably a consequence of ectopic cilia presence. Laboratory blood tests (hemogram, blood urea nitrogen, creatinine, glucose, ALT, AST) were within the normal range for dogs.

Surgery was indicated with the cauterization and excision of the ectopic cilia and third eyelid flap in the right eye. The cauterization of caruncle trichiasis was not authorized by the owner. Was recommended the maintenance of Moxifloxacin ${ }^{2}$ eye drops in the dose of one drop four times a day in both eyes, lubricant based on hyaluronic acid ${ }^{5}$ in the dose of one drop four times a day in both eyes, and autogenous blood serum, obtained from the centrifugation of blood sample of the dog itself, in the dose of one drop every hour in the right eye for $24 \mathrm{~h}$. Meloxicam ${ }^{6}$ in the dose of $0.1 \mathrm{mg} /$ $\mathrm{kg}$ was prescribed every $24 \mathrm{~h}$ for 3 days. Elizabethan collar was recommended until removal of the third eye flap. A sample of eye secretion was collected during surgery and sent for culture and antibiogram and did not show growth of microorganisms.

On return, after 7 days of the surgery procedure, the animal did not show eye discomfort and the corneal ulcer in the right eye was healed. On the 14th day, contact dermatitis was observed in the capton fixation in the skin. The owner was instructed to properly cleansing the area and to apply topical antiseptic based on Chlorhexidine $0.2 \%$. The third eye flap remained stable until the return of $28^{\text {th }}$ day and after removal of the capton, adhesions were identified, and were removed with Castroviejo scissors after application of topical anesthetic based on Proxymetacaine Hydrochloride $^{2}$ eye drops. A fluorescein test was performed and the absence of corneal keratitis was confirmed. Discrete corneal scars (macula) were identified (Figure 1B). Due to exophthalmos it was recommended maintenance of lubricating ${ }^{3,4}$ eye drops every $12 \mathrm{~h}$ until new recommendations and semiannual returns for ophthalmic follow-up. 


\section{DISCUSSION}

The dog presented on this case report was a small breed, male, brachiocephalic breed with exophthalmos, and according with the literature, this one's have greater predisposition to the development of corneal lesions, either due to their facial conformation or changes in eyelid morphology [11]. According to the owner, the dog always showed signs of ocular discomfort caused by superficial corneal ulcers in consequence of ectopic eyelashes but presented an increased pain due to BK $[2,5,8]$.

The patient in this report presented several changes in the eyelid conformation, such as medial corneal entropion, caruncle trichiasis and ectopic cilia, changes that favored recurrent superficial keratitis with consequent development of BK due to corneal decompensation aggravated by metalloproteinases produced by microorganisms.

BK's clinical treatment BK in initial stages includes hyperosmotic solution associated with antibiotics and anti-inflammatory drugs to reduce the lesion [10], however, this treatment alone is not effective in reducing stromal edema and opacity [13], and that underlying causes, such as ectopic cilia must be removed [7], we made the option for the patient's surgical treatment due to the severity of the lesions and the eyelid changes that needed correction so that the cornea would not continue to be damaged.
The choice of surgical technique for the treatment of BK depends on particularities related to the surgeon's preference and the availability of materials and equipment. It was decided to perform a third eyelid flap because it is a low-invasive procedure, that is quickly and easy to perform, and enables the protection and compression of the corneal epithelium which helps in adhesion [3]. This technique has been shown to be effective in the treatment of BK and other eye disorders $[1,10,14,16]$.

The use of topical lubricant based on hyaluronic acid favored corneal reepithelization [12], as well as the maintenance of moxifloxacin, an antimicrobial that is recognized as one of the most effective for the treatment of corneal lesions in dogs, which commonly have Gram microorganisms positive on the ocular surface [15]. Furthermore, the use of autogenous blood serum as an adjuvant in the treatment of diseases favors corneal reepithelization by providing vitamins and growth factors [17].

In this case, surgery and clinical treatment combined resulted in rapid reduction in discomfort of the patient. In general, third eyelid flap is indicated for superficial lesions of the cornea, being maintained for 7 to 15 days [9]. In the present case, was maintained for 28 days in order to allow complete corneal epithelialization, and it is important in these cases to properly cleansing the wound, considering that contact with the surgical thread and capton with the skin can develop corneal lesions. Due to the prolonged time of the third eye flap, adhesions are common, however they are easily

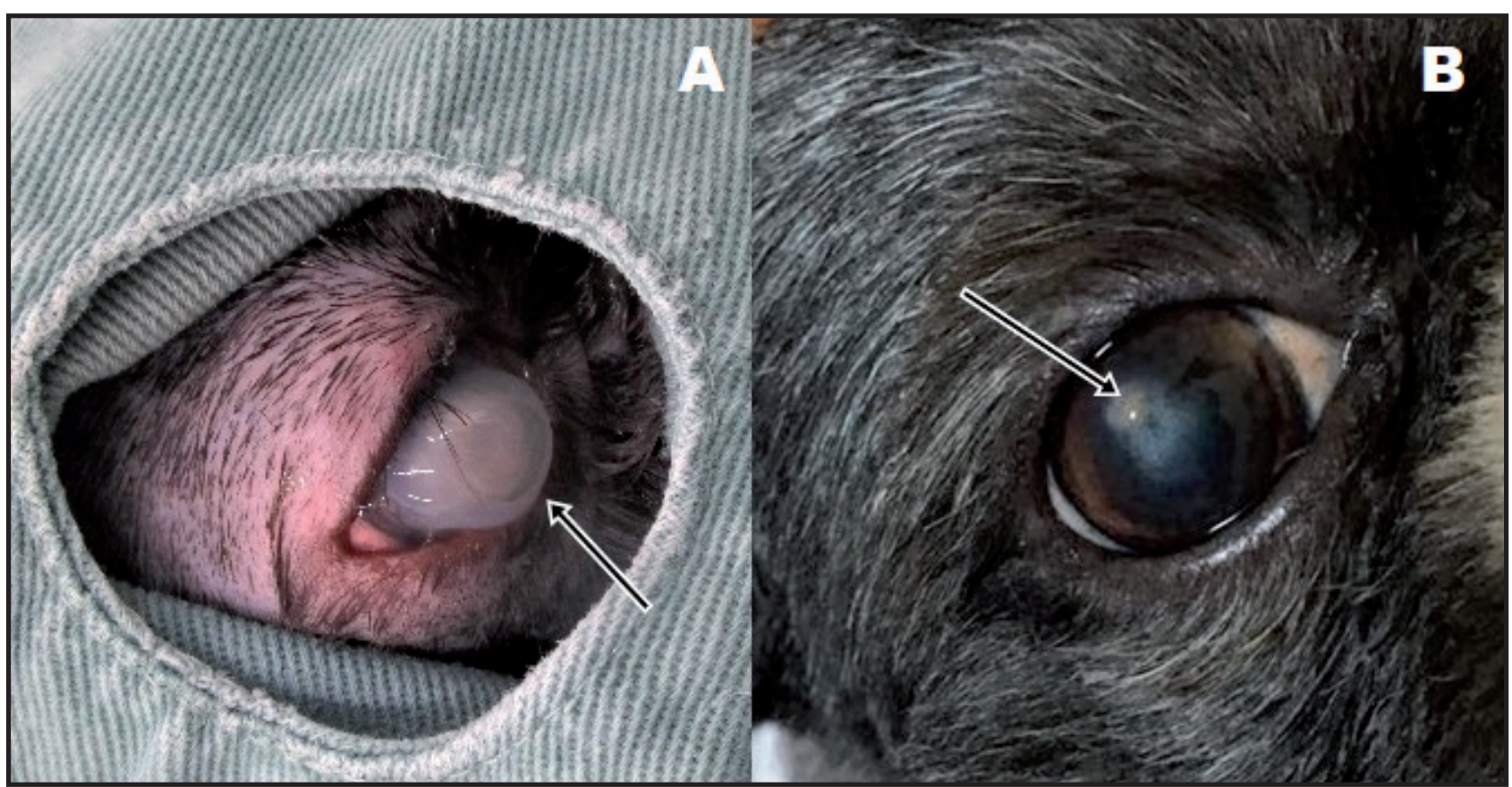

Figure 1. A- BK in a dog. Note the irregular surfaces occupying almost the entire cornea (arrow). B- Postoperative follow up of BK in a dog. Note appearance of the right eye after the end of treatment (28 days) with a negative fluorescein staining and a corneal scar (macula) (arrow). 
removed as performed on the animal reported. Ectopic cilia have been cauterized to eliminate one of the causes of corneal injuries; however, the other alterations could not be corrected because they were not authorized by the owner, which may predispose to the formation of recurrent injuries and a consequent new BK [7].

It is important to emphasize that the covering with the third eyelid maintained for 28 days, associated with the topical use of autogenous blood serum, proved to be effective in reversing the lesions, promoting complete corneal regeneration and recovery of visual function.

\section{MANUFACTURERS}

\author{
${ }^{1}$ Allergan Produtos Farmacêuticos Ltda. Guarulhos, SP, Brazil. \\ ${ }^{2}$ Novartis Biociências S.A. São Paulo, SP, Brazil. \\ ${ }^{3}$ Latinofarma Indústrias Farmacêuticas Ltda. Cotia, SP, Brazil. \\ ${ }^{4}$ Laboratil Farmaceutica Ltda. São Paulo, SP, Brazil. \\ ${ }_{5}^{5}$ Disop S.A. Alcobendas, Madrid, Spain. \\ ${ }^{6}$ Ourofino Saúde Animal Ltda. Cravinhos, SP, Brazil. \\ ${ }^{7}$ Rioquímica S.A. São José do Rio Preto, SP, Brazil.
}

Declaration of interest. The authors report no conflicts of interest. The authors alone are responsible for the content and writing of paper.

\section{REFERENCES}

1 Abreu W.U. \& Philippsen C. 2017. Ceratoplastia com recobrimento de terceira pálpebra em felino doméstico para o tratamento de descemetocele com perfuração de córnea. Acta Scientiae Veterinariae. 45(Suppl 1): 205.

2 Al-Aqaba M., Alomar T., Lowe J. \& Dua H.S. 2011. Corneal nerve aberrations in bullous keratopathy. American Journal of Ophthalmology. 1519(5): 840-849.

3 Cooper S. 2018. Canine superficial chronic corneal epithelial deficits (SCCEDs). Companion animal. 23(6): 342-347.

4 Esson D.W. 2015. Bullous Keratopathy. In: Clinical Atlas of Canine and Feline Ophthalmic Disease. Chichester: John Wiley \& Sons, pp.134-135.

5 Gadelha D.N.B., Cavalcanti B.M., Bravo Filho V., Andrade Júnior N., Batista N.N.C., Escarião A.C.S.L. \& Urbano R.V. 2009. Efeito terapêutico do "cross-linking" corneano na ceratopatia bolhosa sintomática. Arquivo Brasileiro de Oftalmologia. 72(4): 462-466.

6 Gonçalves E.D., Campos M., Paris F., Gomes J.A.P. \& Farias C.C. 2008. Ceratopatia bolhosa: etiopatogênese e tratamento. Arquivos Brasileiros de Oftalmologia. 71(6): 61-64.

7 Hamzianpour N., Lacerda R.P. \& Rhodes M. 2017. Recurrent corneal ulceration in a young dog due to an ectopic cilium in an unusual location. Veterinary Record Case Reports. 5(4): 1-4.

8 Ion L., Boydell P., Ionascu I. \& Bîrtoiu A. 2014. Treatment of bullous keratopathy in the dog. Scientific Works Series C, Veterinary Medicine. 60(1): 58-61.

9 Kwon Y., Kim J., Bae J., Lee K., Kim N. \& Kim M. 2017. Allogeneic tectonic anterior lamellar keratoplasty for the treatment of large corneal perforation in a dog. Pakistan Veterinary Journal. 37(1): 117-119.

10 Oriá A.P., Gomes Junior D.C., Raposo A.C.S., Libório F.A., Schäffer D.P.H. \& Dórea Neto F.A. 2016. Bullous keratopathy in a yellow-headed caracara (Milvago chimachima) treated with a modified third eyelid flap. Pesquisa Veterinária Brasileira. 36(12): 1190-1193.

11 Packer R.M.A., Hendricks A. \& Burn C.C. 2015. Impact of facial conformation on canine health: Corneal ulceration. PLOS ONE. 10(5): 1-16.

12 Pinto-Fraga J., Rosa A.L.R., Arauzo F.B., Rodríguez R.U. \& González-García M.J. 2017. Efficacy and safety of 0.2\% hyaluronic acid in the management of dry eye disease: Eye \& Contact Lens: Science \& Clinical Practice. 43(1): 57-63.

13 Pot S.A., Gallhöfer N.S., Walser-Reinhardt L., Hafezi F. \& Spiess B.M. 2013. Treatment of bullous keratopathy with corneal collagen cross-linking in two dogs. Veterinary Ophthalmology. 18(2):168-173.

14 Ragoggino M., Lamagna B., Guardascione A., Pasolini M.P., Auletta L. \& Lamagna F. 2012. Surgical treatment of feline corneal sequestration using focal keratectomy and third eyelid flap: A retrospective study and literature review. Veterinaria. 26(1): 19-30.

15 Sharma A., Gupta A.K., Singh M. \& Dwivedi1 D.K. 2019. Bacterial investigation and antibiogram in corneal ulcers in dogs. Journal of Animal Research. 9(1): 195-199.

16 Volk H.A., O'Reilly A., Bodley K. \& McCracken H. 2018. Keratomycosis in captive red pandas (Ailurus fulgens): 2 cases. Open Veterinary Journal. 8(2): 200.

17 Von Hofsten J., Egardt M.\& Zetterberg M.2016. The use of autologous serum for the treatment of ocular surface disease at a Swedish tertiary referral center. International Medical Case Reports Journal. 9: 47-54. 